\title{
The way irrelevant dimensions are processed depends on what they overlap with: The case of Stroop- and Simon-like stimuli
}

\author{
Sylvan Kornblum \\ Mental Health Research Institute, The University of Michigan, 205 Zina Pitcher Place, Ann Arbor, MI 48109-0720, U.S.A.; \\ e-Mail: SYLVAN.KORNBLUM@umich.edu
}

Summary. According to the dimensional-overlap model (Kornblum, 1992), irrelevant dimensions that overlap with a stimulus dimension (e.g., Stroop-type stimuli) are processed by a different stage than those that overlap with the response (e.g., Simon-type stimuli). We show that the effects of these two types of overlap are additive, thus supporting the model's hypothesis. We also show that the time course of facilitation and interference is different for these two types of overlap.

\section{Introduction}

The dimensional-overlap model

A model has recently been proposed (Kornblum, 1992; Kornblum, Hasbroucq, \& Osman, 1990) for stimulusstimulus (S-S) and stimulus-response (S-R) compatibility that lays the foundations for a taxonomy in which the various components of simple and relatively complex compatibility tasks (e.g., the Simon and the Stroop tasks) can be identified and compared. According to this model, compatibility effects are due, in part, to the dimensional overlap of the stimulus and the response sets, and/or of the stimulus sets in the task. We define dimensional overlap as the degree to which sets of items are perceptually, structurally, or conceptually similar. Dimensional overlap is thus a property of the mental representations of sets and is heir to all the strengths and weaknesses inherent in the concept of similarity. It follows from the processing assumptions of the model that dimensional overlap affects performance whether the overlapping dimensions are relevant or not.

Dimensional overlap and dimensional relevance are the two factors used to generate the eight-class taxonomy of the model (see Table 1). The processing component of the model makes specific predictions about the compatibility effects in these classes. In a number of cases these pre- dictions have already been confirmed either in our own laboratory and/or in the literature (see Kornblum, 1992).

\section{The taxonomy}

Type-1 ensembles are characterized by the absence of dimensional overlap in either the relevant or the irrelevant dimensions. Examples of Type 1 ensembles are ubiquitous in the choice-RT literature. If, as the model asserts, compatibility requires dimensional overlap, it follows that Type-1 ensembles do not produce compatibility effects. However, such ensembles have been quite useful in constructing neutral, baseline, control conditions for the study of such effects.

Type- 2 ensembles are characterized by overlap between the response and the relevant stimulus dimensions of the $\mathrm{S}-\mathrm{R}$ ensemble. These are the classical ensembles that are ordinarily used in the study of stimulus-response compatibility (see, Fitts \& Deininger, 1954).

Type-3 ensembles are characterized by having the only overlap occurring between the response set and the irrelevant stimulus dimension. This is the type of ensemble that produces the so-called Simon effect, strictly defined (e.g., Simon \& Small, 1969; see also Kornblum, 1992, p. 763, fn. 7).

Type-4 ensembles have the only dimensional overlap occurring between the relevant and irrelevant stimulus dimension. This type of ensemble characterizes all the Stroop-like tasks that have tried to preserve Stroop-like characteristics in the stimulus properties of the task (e.g., Keele, 1972), and dissociate their effects from those of the response.

Type-5 ensembles are infrequent. The best-known example is that originally studied and published by Hedge and Marsh (1975), and requires a two-dimensional response set in which one dimension overlaps with the relevant stimulus dimension, the other with the irrelevant stimulus dimension, while the two dimensions themselves do not overlap with each other. 
Table 1. A taxonomy of $S-R$ ensembles, illustrating the various combinations of dimensional overlap with relevant and/or irrelevant dimensions of the stimulus and/or response, with some examples of such ensembles from the literature

\begin{tabular}{|c|c|c|c|c|}
\hline \multirow{3}{*}{$\begin{array}{l}\text { Ensemble } \\
\text { type }\end{array}$} & \multicolumn{3}{|c|}{ Overlapping ensemble dimensions } & \multirow{3}{*}{$\begin{array}{l}\text { Representative } \\
\text { studies }\end{array}$} \\
\hline & \multicolumn{2}{|c|}{$\begin{array}{l}\text { Stimulus-response } \\
\text { dimensions }\end{array}$} & \multirow{2}{*}{$\begin{array}{l}\text { Stimulus- } \\
\text { stimulus } \\
\text { dimensions }\end{array}$} & \\
\hline & relevant & irrelevant & & \\
\hline 1 & no & no & no & $\begin{array}{l}\text { Many choice RT } \\
\text { tasks that have no } \\
\text { dimensional overlap }\end{array}$ \\
\hline 2 & yes & no & no & $\begin{array}{l}\text { Fitts \& Seeger, 1953; } \\
\text { Fitts \& Deininger, } \\
1954\end{array}$ \\
\hline 3 & no & yes & no & $\begin{array}{l}\text { Simon, } 1969 ; \\
\text { Wallace, } 1971\end{array}$ \\
\hline 4 & no & no & yes & $\begin{array}{l}\text { Keele, 1972; } \\
\text { Kahneman \& } \\
\text { Henick, 1981; } \\
\text { Ericksen \& Ericksen, } \\
1974\end{array}$ \\
\hline 5 & yes & yes & no & Hedge \& Marsh, 1975 \\
\hline 6 & yes & no & yes & (None) \\
\hline 7 & no & yes & yes & $\begin{array}{l}\text { (See composite } \\
\text { condition in the } \\
\text { present study) }\end{array}$ \\
\hline 8 & yes & yes & yes & $\begin{array}{l}\text { Stroop, 1935; Simon } \\
\text { \& Rudell, } 1967\end{array}$ \\
\hline
\end{tabular}

Type- 6 ensembles require three-dimensional stimuli and one-dimensional responses with the following overlap pattern: the relevant stimulus dimension is the only dimension that overlaps with the response; there is no overlap between the response and any irrelevant dimension; however, there is overlap between two stimulus dimensions. These constraints and requirements lead to a three-dimensional stimulus in which the two irrelevant dimensions overlap with each other only. To the best of my knowledge, no studies have been done with this type of ensemble.
Type-7 ensembles also require three-dimensional stimuli and one-dimensional responses. Here, the relevant stimulus dimension does not overlap with the response; however, it does overlap with an irrelevant stimulus dimension. Concurrently, there is a third stimulus dimension (irrelevant) that does not overlap with either of the previous two stimulus dimensions, but does overlap with the response. To the best of my knowledge, the composite S-S/S-R condition in the present study is the only published experiment with this type of ensemble.

Type- 8 ensembles are characterized by having the response set overlap with both the relevant and the irrelevant stimulus dimensions which themselves overlap. This dimension is necessarily one and the same everywhere. This type of ensemble is probably the best known in that it encompasses the standard Stroop task and others like it (see MacCleod, 1991, for a review).

\section{Processing Type-3, Type-4, and Type-7 ensembles}

The model postulates that if the stimulus and response sets in an S-R ensemble have dimensions that overlap (e.g. Type- 2 ensembles), the presentation of an element from the stimulus set automatically activates its corresponding element in the response set. This process is represented by the upper branch of the response-production stage as shown in Figure 1. Before this response can be executed, however, the correct response must be identified. This process is represented by the lower branch of the response-production stage. Response identification may occur in one of three ways: by use of the identity rule, by use of another rule, or by a search. The time required for response identification is shortest with the identity rule, longest with the search, and usually intermediate with other rules, depending on their complexity. If the automatically activated and the correct responses do not differ, the response so identified is executed without further ado. But if these two responses do differ, then the automatically activated response is aborted, the program for the correct response retrieved, and the response is then executed. Automatic activation of the re-

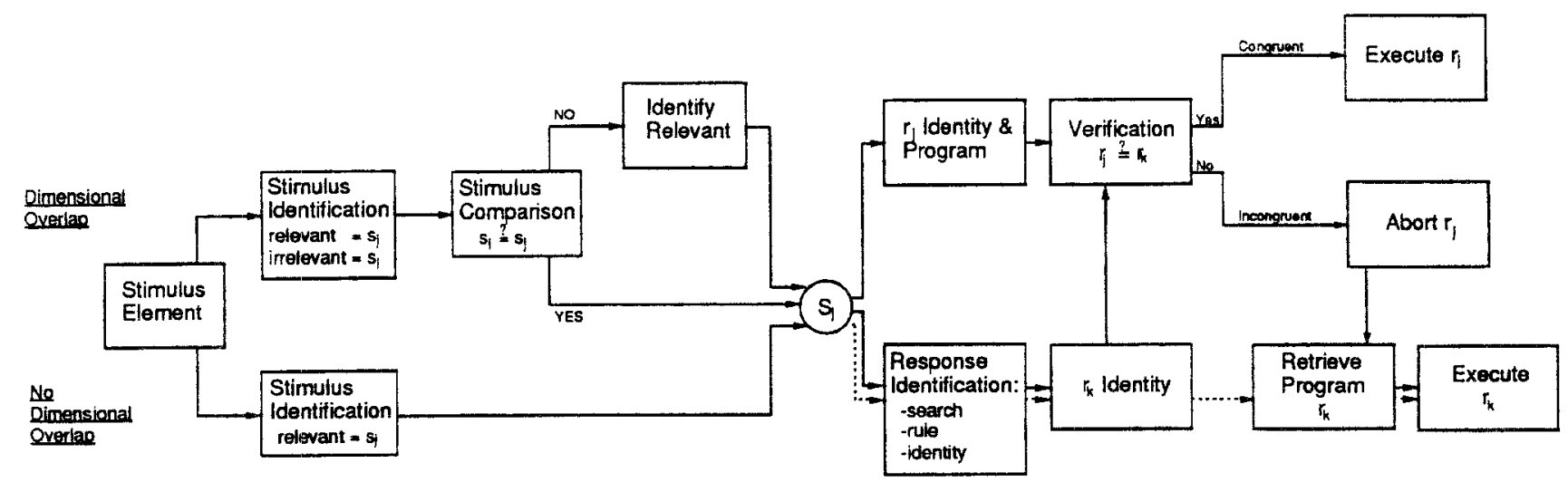

Fig. 1. Processing network of the dimensional-overlap model. The circle with an $\mathrm{S}_{\mathrm{j}}$ in the center represents a stimulus vector and marks the cut-point in the network (see Schweickert, Fisher, \& Goldstein, 1991) 
sponse occurs whether the overlap is with the relevant (e. g., Type-2 ensemble) or with an irrelevant (e.g., Type-3 ensemble) stimulus dimension.

If, in contrast to the above case, no dimension of the stimulus overlaps with the response, but the relevant and irrelevant dimensions of the stimulus do overlap (e.g., Type- 4 ensemble), then the presentation of a stimulus element automatically activates two stimulus-identification codes as potential candidates for tagging as the relevant dimension. If the two codes or features do not differ, then it matters little which is tagged, and the stimulus-attribute vector with this tagged attribute ( $\mathrm{S}$ in Figure 1$)$ is passed on to the response-production stage. If the two codes do differ, then one of them is selected and tagged as relevant before being passed on to the response-production stage. It is on the basis of the tagged attribute that the correct response is subsequently identified.

The effects of irrelevant stimulus dimensions in Type-3 and Type- 4 ensembles are thus seen to be mediated by two separate stages: the response-production stage for Type-3, and the stimulus-identification stage for Type- 4 . If these overlap characteristics are now combined in a Type-7 ensemble (see Table 1), performance with Type-7 ensembles should be predictable from the individual performances with Type- 3 and Type- 4 ensembles, i.e., they should be additive (Sternberg, 1969). The purpose of the present study was to test these predictions and to explore the timecourse properties of these processes.

\section{Method}

\section{Stimuli and responses}

The stimuli consisted of colors presented as the background for either words or a five-letter string inside a white rectangular frame in the center of a CRT screen ( $640 \times 480$ pixels). The frame was 87 pixels long and 35 pixels high, or approximately $3.2 \times 1.2 \mathrm{~cm}$. The words and the letter string were centered in the rectangle. The color background filled the entire rectangle during training; at other times the colors were presented in the left, right, upper, or lower half of the rectangle, depending on the experimental conditions.

The rectangle in which the stimuli appeared was defined by a white border, 1 pixel wide. The colored and uncolored halves of the rectangle were separated by a white line, 1 pixel wide. The words were written in white capital letters edged in black lines, 1 pixel wide. The unused portion of the CRT screen, as well as the uncolored half of the stimulus rectangle, were both black.

There were two colors: blue and green; four words: BLUE, GREEN, NOVEL, and ELBOW; one five-letter string: FGHSV; and one achromatic gray.

The responses were key presses made with the index finger of the right or left hand. The relevant stimuli were the colors blue and green. The words, the letter string, and the position of the colors inside the rectangle were all irrelevant dimensions of the task; i.e., subjects were instructed to ignore them, and they had a correlation of zero with the correct response.

\section{Primes}

The primes in this study consisted of advance information concerning the value of the irrelevant dimensions in a trial. For irrelevant words, the word was presented in the stimulus rectangle before the color was.
For irrelevant positions, that half of the stimulus rectangle in which the color would eventually appear was filled with gray, which was subsequently replaced by the color. Both irrelevant dimensions, word and position, were present in all trials and were displayed in the manner just described, before the color.

\section{Trial types}

In addition to training trials, there were four types of trial defined by the nature of the overlap of the irrelevant dimension. The irrelevant dimension(s) could overlap with the stimulus (S-S overlap), the response (S-R overlap), both (S-S/S-R overlap), or neither (neutral).

Training. The training stimuli consisted of the colors blue or green filling the entire stimulus rectangle with the five-letter string FGHSV in the foreground.

Neutral trials (Type 1). The colors were presented in the upper or lower half of the rectangle; the irrelevant words were NOVEL or ELBOW. This combination produced four different kinds of trial, all of which were neutral with respect to the relevant stimuli (colors) and the responses (left/right key presses) - we call these neutral trials.

Simple $S$-R overlap trials (Type 3). The colors were presented in the left or right half of the rectangle; the irrelevant words were NOVEL or ELBOW. This combination produced four different kinds of trial: two in which the side of the color matched the side of the response - we call these $S-R$ consistent trials; and two in which the side of the color conflicted with the side of the response - we call these $S-R$ inconsistent trials.

Simple $S-S$ overlap trials (Type 4). The colors were presented in the upper or lower half of the rectangle; the irrelevant words were BLUE or GREEN. This combination produced four different kinds of trial: two in which the word matched the color - we call these $S$-S consistent trials, and two in which the word conflicted with the color - we call these $S$-S inconsistent trials.

Composite S-S/S-R overlap trials (Type 7). The colors were presented in the left or right half of the rectangle; the irrelevant words were BLUE or GREEN. This combination produced eight different kinds of trial: two in which the color word matched the color, and the side of the color matched the side of the response - we call these $S-S / S-R$ consistent trials; two in which the color word conflicted with the color, and the side of the color conflicted with the side of the response - we call these $S-S / S-R$ inconsistent trials; two trials in which the word matched the color and the side conflicted with the response, and two trials in which the word conflicted with the color, but the side matched the response - we call these $S-S / S-R$ hybrid trials.

\section{Trial events}

Each trial began with a warning signal consisting of the four white corner lines of the stimulus rectangle. After a randomly selected interval of between 400 and $600 \mathrm{~ms}$, either the full stimulus (lag-zero condition), or a prime (lag-200 condition) was presented. In either case the four corners of the warning signal were incorporated into the white frame of the stimulus rectangle. If a prime had occurred at that point in the trial sequence, then the stimulus color was presented $200 \mathrm{~ms}$ after the onset of the prime. The stimulus display was terminated by the subject's key-press response, and this was followed by feedback which was presented on the screen for $1.5 \mathrm{~s}$, and included the score that the subject had earned on this trial, as well as information on whether or not he had made an error. After a randomly selected interval of between 600 and 1,200 ms following the termination of the feedback, the warning signal for the next trial appeared on the screen. 
Table 2. Mean reaction times, standard deviations, error rates, and consistency effects for the simple S-S, S-R, and neutral conditions

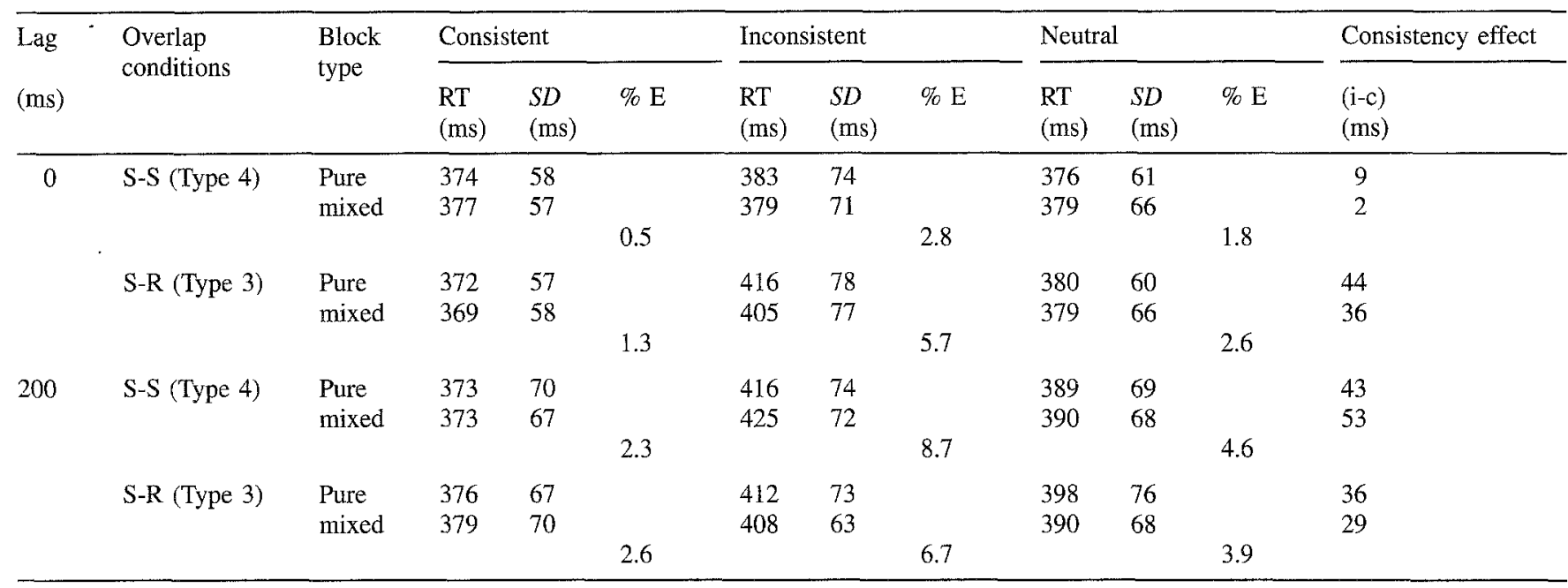

The error percentages are averaged over mixed and pure blocks. The consistency effects are the differences between inconsistent and consistent RTs

\section{Experimental conditions}

Conditions were run in blocks of 72 trials. There were four principal experimental conditions, plus a training condition, all defined by the trial composition of a block.

Training-block. The training blocks consisted of the equiprobable presentation of the two training stimuli.

Simple S-S overlap, pure block. Simple S-S overlap blocks consisted of one-third S-S consistent trials, one-third S-S inconsistent trials, and one-third neutral trials.

Simple S-R overlap, pure block. Simple S-R overlap blocks consisted of one-third S-R consistent trials, one-third S-R inconsistent trials, and one-third neutral trials.

Simple $S-S$ and $S$-R overlap, mixed blocks. Mixed blocks consisted of one-sixth $S-S$ consistent trials, one-sixth $S-R$ consistent trials, onesixth S-S inconsistent trials, and one-sixth S-R inconsistent trials. Onethird of the trials were neutral.

Composite $S-S / S-R$ overlap. $S-S / S-R$ overlap blocks consisted of onesixth S-S/S-R consistent trials, one-sixth S-S/S-R inconsistent trials, and one-third S-S/S-R hybrid trials, with half of those being S-S consistent and half S-S inconsistent; another third of the trials in these blocks were neutral.

\section{Design}

The experiment lasted for one 2-hour session. All subjects started with two training blocks. Each of the subsequent four experimental conditions was run with lag-zero and lag-200 $\mathrm{ms}$. The lag conditions were blocked and run back to back. The four experimental conditions were arranged in two Latin Squares with eight subjects having the lag-zero conditions first, and another eight subjects having the lag-200 conditions first. Half of the subjects received one mapping of colors to keys, the other half received the other mapping; mapping was balanced over the other conditions.

The simple pure S-S overlap and S-R overlap conditions were each run for one block of 72 trials with a break at the 36th trial point. The simple mixed S-S overlap and S-R overlap conditions, as well as the composite S-S/S-R overlap conditions, were each run for two 72-trial blocks, and also with a break at the 36th trial point for each block. When a new condition was begun, 16 warm-up trials preceded the experimental block.

\section{Subjects, procedures and equipment}

Sixteen subjects, recruited by advertisement in the student newspaper were run. They were right handed native English-speaking males with no visual, auditory, or motor handicaps. As part of the screening procedure all subjects were required to pass the Ishihara color-blindness test.

Subjects were run in a dimly illuminated room, and sat $75 \mathrm{~cm}$ away from the CRT screen on which the stimuli were displayed. The keys required a displacement of $3-4 \mathrm{~mm}$ and a force of $30 \mathrm{gm}$ to be activated. A payoff matrix that weighed speed and accuracy differentially enabled subjects to earn bonuses based on their performance.

Stimuli were displayed on a NEC, multisync, 3D, 640×480 super VGA monitor. The experiment was controlled by a 386 PC.

\section{Results}

\section{Simple S-S and S-R overlap, pure and mixed Blocks}

The mapping of color to key-presses was not a significant factor in this experiment, $F(1,14)=2.89, p=.1113$, and is therefore dropped from further consideration. The pureand mixed-block factor likewise did not have a significant effect $F(1,15)=0.2, p=.8773$, for either S-S overlap, $F(1,15)=.2, p=.66$, or S-R overlap, $F(1,15)=1.77, p=.2$, conditions and is also dropped from subsequent analyses.

The difference between consistent and inconsistent trials (the consistency effect) was significant overall $(32 \mathrm{~ms}$, $F(1,15)=74.91, p<.0001$. However, consistency interacted with lag and overlap conditions, $F(1,15)=32.22$, $p<.0001$. Consistency had no significant effect for S-S overlap conditions at lag zero $(5 \mathrm{~ms}), F(1,15)=1.7$, $p=.2124$; however, it did have a significant effect $(48 \mathrm{~ms})$ at lag $200, F(1,15)=28.83, p<.0001$. For the S-R overlap condition, consistency had a significant effect $(40 \mathrm{~ms})$ at lag zero, $F(1,15)=63.13, p<.0001$, and a reduced (32 ms), 


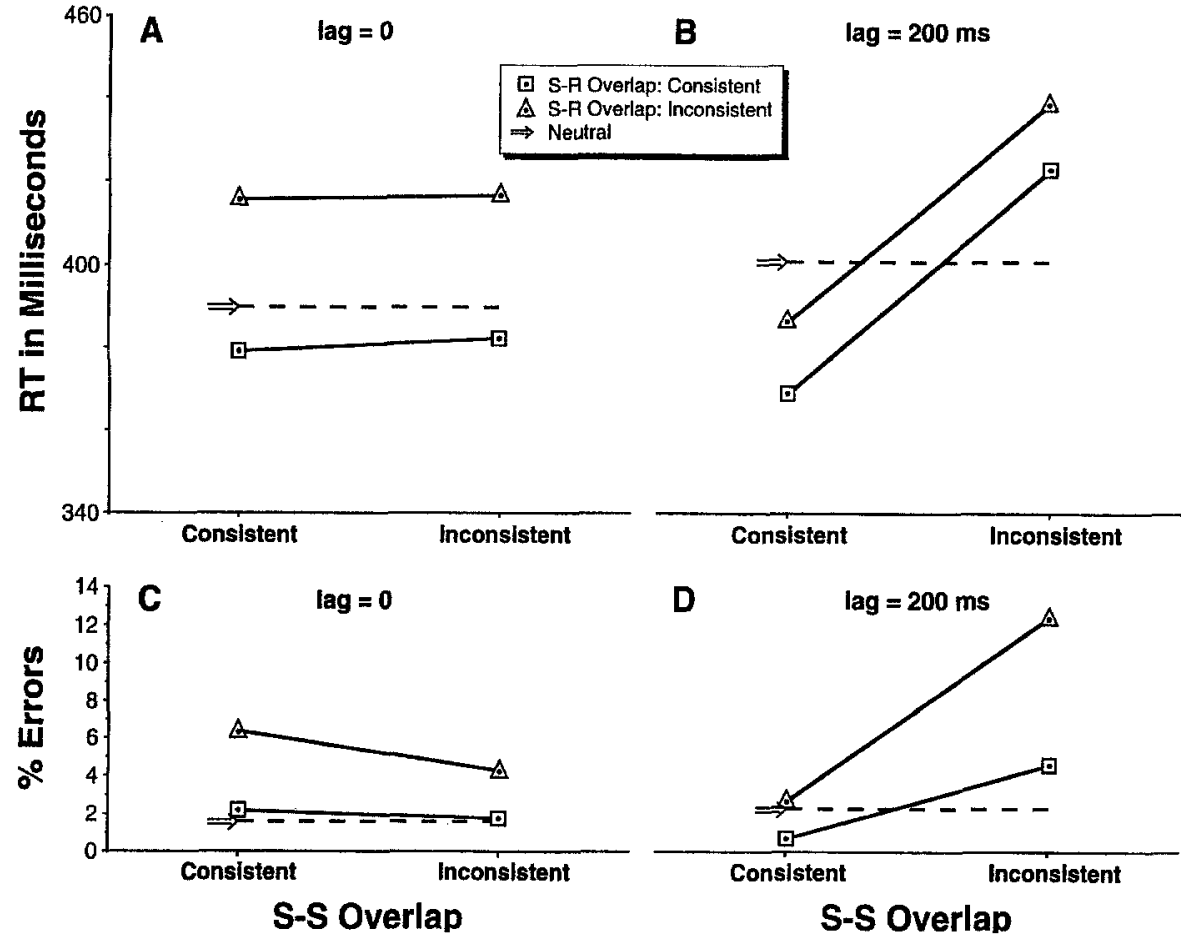

Fig. 2. Mean reaction times and errors for the composite S-S/S-R conditions at lag zero (panels A and C) and lag 200 (panels B and D) but still significant, effect at lag $200, F(1,15)=69.36$, $p<.0001$. The context, (S-S or S-R overlap) had no significant effect on the neutral condition $F(1,15)=3.65$, $p=.0753$; however, lag did have a small $(13 \mathrm{~ms})$, but significant, effect, $F(1,15)=7.73, p<.0149$, on neutral trials.

\section{Composite $S-S / S-R$ overlap conditions}

At lag zero consistency did not have a significant effect for $S-S$ overlap, however, it did have a significant effect (36 ms) for S-R overlap, $F(1,15)=25.75 ; p<.0001$. At lag 200 consistency had a significant effect for both S-S overlap (53 ms), $F(1,15)=107.44, p<.0001$, and S-R overlap $(17 \mathrm{~ms}), F(1,15)=11.23, p<.004$. There was also a significant difference in the consistency effect between lag zero and lag 200 for S-S overlap $(51 \mathrm{~ms})$, $F(1,15)=74.57, p<.0001$, and for S-R overlap $(-19 \mathrm{~ms})$, $F(1,15)=8.9, p<.009$. Consistency did not interact with overlap at either lag zero, $F(1,15)=0.06, p=.8042$, or lag $200, F(1,15)=0.35, p=.5645$. Lag had a small $(12 \mathrm{~ms})$, but significant, effect on the neutral condition, $F(1,15)=6.37$, $p<.0234$. Context (simple vs. composite) similarly had a small $(10 \mathrm{~ms})$, but significant, effect, $F(1,15)=17.4$, $p<.0008$, on the neutral condition.

\section{Discussion}

It is clear from these results that irrelevant stimulus dimensions have different processing consequences, depending on whether they overlap with the response (Type 3 ) or with a relevant stimulus dimension (Type 4). These different effects interact with lag. Consider the simple S-S and S-R overlap conditions (Types 4 and 3, respectively). At lag zero the consistency effect for S-S overlap fails to reach statistical significance. However, when lag is increased to $200 \mathrm{~ms}$, a highly significant 48 -ms consistency effect emerges. For S-R overlap the consistency effect is significant at both lag zero and lag 200. A similar pattern is obtained with the composite (Type 7) condition: at lag zero the consistency effect for S-S overlap is nonexistent $(2 \mathrm{~ms})$. However, when lag is increased to $200 \mathrm{~ms}$, a highly significant 53-ms-consistency effect is found. For S-R overlap a highly significant consistency effect is obtained at both lag zero and lag 200. As was the case for the simple S-S and S-R overlap conditions, the consistency effect for S-R overlap decreases with increasing lag. At this point we may conclude that the processing of Type- 3 and Type- 4 ensembles each has a different time course. However, this does not imply that they are processed by different stages. To address this question, we turn to the composite S-S/S-R condition (Type 7).

Because instances of consistent and inconsistent trials for Type-3 and Type- 4 ensembles were factorially combined within trials to form the Type 7, composite S-S/S-R overlap condition, the data from this condition permit us to assess the additivity of the overlap factor effects (S-S or S-R) on the consistency effect. These data are shown in Figure 2. Here we see that at lag zero the S-R consistency effect is $36 \mathrm{~ms}$, irrespective of S-S consistency; at lag 200 the S-R consistency effect has dropped to $17 \mathrm{~ms}$, while the $\mathrm{S}-\mathrm{S}$ consistency effect is now $53 \mathrm{~ms}$. These effects are additive. The magnitude of the consistency effects in the composite (Type 7) conditions are identical, with one exception, to those obtained in the simple mixed Type- 3 and Type- 4 conditions (see Table 2), as would be expected from 
the model: the consistency effect for S-S overlap at lag zero is $2 \mathrm{~ms}$ for both simple and composite conditions; for S-S overlap at lag $200 \mathrm{it}$ is $53 \mathrm{~ms}$ for both simple and composite conditions; for S-R overlap at lag zero it is $36 \mathrm{~ms}$, again for both conditions; and finally for S-R overlap at lag 200 it is $36 \mathrm{~ms}$ for the simple, and $17 \mathrm{~ms}$ for the composite, conditions. Together, these data provide strong support for the assertion of the dimensional overlap model that irrelevant stimulus dimensions that overlap with the response (Type 3 ) are processed differently, and by a different stage, than those that overlap with the stimulus (Type 4).

Lag and the simple/composite factor each had small, significant, and additive effects on the RT for the neutral condition. The effect of lag is $12 \mathrm{~ms}$ on average (13 ms for the simple and $11 \mathrm{~ms}$ for the composite conditions). The effect of simple/composite is $10 \mathrm{~ms}$ on average $(11 \mathrm{~ms}$ at lag zero and $9 \mathrm{~ms}$ at lag 200). These two effects are examined next.

Recall that there was no significant difference between pure and mixed blocks. Contrast this with the small, but significant, difference between simple and composite conditions. At least two possible reasons for this difference seem plausible: either it was perceptually more difficult to identify the relevant stimulus in a composite trial than it was in either a mixed or a pure block trial, or it was easier to switch between the different trial types (see Allport, Styles, \& Hsieh, in press) in the pure and mixed blocks than it was in the composite blocks. This issue cannot be resolved from the present data.

The second finding concerns the effect of lag on the neutral condition (where the RT for neutral conditions is $12 \mathrm{~ms}$ longer at lag 200 than at lag zero). This effect is present for both simple and composite conditions. One possible, and reasonable, explanation for this finding would be to attribute the difference to paracontrast (forwardmasking) effects of the primes at lag 200 (see Breitmeyer, 1984). This hypothesis has interesting implications. First, if the primes exerted a masking effect on the neutral condition, they in all likelihood exerted the same effect on the overlapping conditions. If one assumes this to be the case, then the consistency effects may be partitioned into facilitation and interference components by simply taking the difference between the appropriately matched neutral RTs and the consistent and the inconsistent RTs, respectively. These subtractions show (see Table 2) that for Type-3 ensembles (S-R overlap), the bulk of the consistency effect at lag zero is comprised of interference ( $9 \mathrm{~ms}$ vs. $31 \mathrm{~ms}$ ) with facilitation and interference becoming roughly equal $(17 \mathrm{~ms}$ vs. $16 \mathrm{~ms}$ ) at lag 200 . For Type- 4 ensembles, there is no consistency effect at lag zero, hence nothing to partition. However, at lag 200 the facilitation effect is roughly half the size of the interference effect ( $17 \mathrm{~ms}$ vs. $31 \mathrm{~ms})$, and the facilitation effect for Type 3 and Type 4 are approximately equal. While these interpretations are, of course, tentative and require verification, they are consistent with the overall picture suggested by this study, that Type- 3 and Type- 4 ensembles appear to have very distinct and separate processing paths with additive consequences.
Acknowledgements. Funding for this research was provided in part by NIMH grant MH 43287 and NSF grant DBS 9222324.

I thank Tony Whipple for technical assistance and support, as well as many insightful comments, Bridget Smith for collecting the data, and Erick Lauber and Huazhong Zhang for many useful discussions.

\section{References}

Allport, A., Styles, E. A., \& Hsieh, S.-L. (in press) Non-spatial orienting of attention: Exploring the dynamic control of tasks. In $\mathrm{C}$. Umilta \& M. Moscovitch (Eds.), Attention and performance XV. Cambridge, MA: MIT Press.

Breitmeyer, B. G. (1984) Visual masking: An integrative approach. New York Oxford University Press.

Ericksen, B. A., \& Ericksen, C. W. (1974) Effects of noise letters upon the identification of a target letter in a nonsearch task. Perception \& Psychophysics, 16, 143-149.

Fitts, P. M., \& Deininger, R. L. (1954) S-R compatibility: Correspondence among paired elements within stimulus and response codes. Journal of Experimental Psychology, 48, 483-492.

Fitts, P. M., \& Seeger, C. M. (1953) S-R compatibility: Spatial characteristics of stimulus and response codes. Joumal of Experimental Psychology, 46, 199-210.

Hedge, A., \& Marsh, N. W. A. (1975) The effect of irrelevant spatial correspondence on two-choice response-time. Acta Psychologica, $39,427-439$.

Kahneman, D., \& Henick, A. (1981) Perceptual organization and attention. In M. Kubovy \& J. R. Pomerants (Eds.), Perceptual organization (pp. 181-211). Hillsdale, NJ: Erlbaum.

Keele, S. W. (1972) Attention demands of memory retrieval. Journal of Experimental Psychology, 93, 245-248.

Kornblum, S. (1992) Dimensional overlap and dimensional relevance in stimulus-response and stimulus-stimulus compatibility. In G. E. Stelmach \& J. Requin (Eds.), Tutorials in motor behavior II (pp. 743-777). Amsterdam, Netherlands: Elsevier Science Publishers.

Kornblum, S., Hasbroucq, T., \& Osman, A. (1990) Dimensional overlap: Cognitive basis for stimulus-response compatibility - A model and taxonomy. Psychological Review, 97, 253-270.

MacLeod, C. (1991) Half a century of research on the Stroop effect: An integrative review. Psychological Bulletin, 109, 163-203.

Schweickert, R., Fisher, D. L., \& Goldstein, W. M. (1991). General latent network theory: Structural and quantitative analysis of networks of cognitive processes. Manuscript, Department of Psychology, Purdue University, West Lafayette, Indiana.

Simon, J. R. (1969) Reactions toward the source of stimulation. Journal of Experimental Psychology, 81, 174-176.

Simon, J. R., \& Rudell, A. P. (1967). Auditory S-R compatibility: The effect of an irrelevant cue on information processing. Journal of Applied Psychology, 51, 300-304.

Simon, J. R., \& Small, A. M. (1969). Processing auditory information: Interference from an irrelevant cue. Journal of Applied Psychology, $53,433-435$.

Sternberg, S. (1969). The discovery of processing stages. Extensions of Donders' method. In W. G. Koster (Ed.), Attention and performance II: Proceedings from a Symposium on Attention and Performance (Special issue). Acta Psychologica, 30, 276-315.

Stroop, J. R. (1935) Studies of interference in serial verbal reactions. Journal of Experimental Psychology, 18, 643-662.

Wallace, R. J. (1971) S-R compatibility and the idea of a response code. Joumal of Experimental Psychology, 88, 354-360. 